\title{
CONVERGENCE TO NONLINEAR DIFFUSION WAVES FOR SOLUTIONS OF THE INITIAL BOUNDARY PROBLEM TO THE HYPERBOLIC CONSERVATION LAWS WITH DAMPING
}

\author{
BY \\ PIERANGElo MARCATI (Dipartimento di Matematica Pura ed Applicata, Università degli Studi \\ di L'Aquila, Italy) \\ AND \\ MING MEI (Department of Mathematics, Faculty of Science, Kanazawa University, Japan)
}

\begin{abstract}
In this paper we consider a model of hyperbolic balance laws with damping on the quarter plane $(x, t) \in \mathbb{R}_{+} \times \mathbb{R}_{+}$. By means of a suitable shift function, which will play a key role to overcome the difficulty of large boundary perturbations, we show that the IBVP solutions converge time-asymptotically to the shifted nonlinear diffusion wave solutions of the Cauchy problem to the nonlinear parabolic equation given by the related Darcy's law. We obtain also the time decay rates, which are the optimal ones in the $L^{2}$-sense. Our proof is based on the use of the classical energy method.
\end{abstract}

1. Introduction. Let us consider the following model of hyperbolic equations with damping, on the quarter plane $\mathbb{R}_{+} \times \mathbb{R}_{+}\left(\mathbb{R}_{+}=(0,+\infty)\right)$ given by

$$
\left\{\begin{array}{l}
v_{t}-u_{x}=0, \\
u_{t}+p(v)_{x}=-\alpha u,
\end{array} \quad(x, t) \in \mathbb{R}_{+} \times \mathbb{R}_{+},\right.
$$

which models a compressible flow with dissipative external force field in Lagrangian coordinates. The external force term $-\alpha u$ appears in the momentum equation. Here, $v>0$ is the specific volume, $u$ is the velocity, the pressure $p(v)$ is a smooth function of $v$ such that $p(v)>0, p^{\prime}(v)<0$, and $\alpha>0$ is the damping constant.

It has been proved in Marcati and Milani [13] in the case of weak solutions and in Hsiao and Liu [7], Nishihara [19] in the case of smooth solutions that the solutions $(v, u)(x, t)$ to the corresponding Cauchy problem of (1.1) tend time-asymptotically to the nonlinear self-similar diffusion wave solutions $(\bar{v}, \bar{u})(x, t)(\bar{v}(x, t)=\phi(x / \sqrt{1+t}))$ of the porous

Received September 30, 1998.

2000 Mathematics Subject Classification. Primary 35L65; Secondary 35B40, 76R50.

E-mail address: marcati@univaq.it

E-mail address: mei@kappa.s.kanazawa-u.ac.jp

(C)2000 Brown University 
media equation

$$
\left\{\begin{array}{l}
\bar{v}_{t}=-\frac{1}{\alpha} p(\bar{v})_{x x}, \\
p(\bar{v})_{x}=-\alpha \bar{u},
\end{array} \quad(x, t) \in \mathbb{R} \times \mathbb{R}_{+},\right.
$$

namely

$$
\left\{\begin{array}{l}
\bar{v}_{t}-\bar{u}_{x}=0, \\
p(\bar{v})_{x}=-\alpha \bar{u},
\end{array} \quad(x, t) \in \mathbb{R} \times \mathbb{R}_{+} .\right.
$$

The convergence theory on the nonlinear diffusion waves for the Cauchy problem can be found, for instance, in $[14,3,1,8,2,9,5,4,15]$, and in the references quoted in those papers.

Denote by $\bar{v}(x, t)$ any solution of (1.2) with the end states

$$
\bar{v}( \pm \infty, t)=v_{ \pm}, \quad v_{+} \neq v_{-} .
$$

Due to the Darcy law $\bar{u}(x, t)=-\frac{1}{\alpha} p(\bar{v})_{x}$, we have

$$
\bar{u}( \pm \infty, t)=0 \text {. }
$$

Suppose that the initial data for (1.1) satisfy the following limiting conditions:

$$
\left.(v, u)\right|_{t=0}=\left(v_{0}, u_{0}\right)(x) \rightarrow\left(v_{+}, u_{+}\right) \quad \text { as } x \rightarrow+\infty .
$$

Moreover, we assume that the following boundary condition for (1.1) holds:

$$
\left.v\right|_{x=0}=g(t), \quad t \in \mathbb{R}_{+},
$$

where $g(t)$ takes a value on $\left[v_{+}, v_{-}\right]$(or say $\left[v_{-}, v_{+}\right]$, if $v_{-}<v_{+}$). This kind of boundary condition arises in several physical problems and, in particular, it has been considered, in a different setting, to model the isentropic hydrodynamic flow of electrons in a semiconductor device, where the Ohmic contact is described by using a boundary condition on the electron density. This problem will be considered in a forthcoming paper by the authors.

The main purpose of this paper is to show that the solutions of (1.1) with the initial data (1.6) and the boundary condition (1.7) converge to the nonlinear diffusion wave solutions of (1.2) when $v_{+} \neq v_{-}$and the initial-boundary perturbations are small. This will be given in the following Sections 2 and 3 . In the special case $v_{+}=v_{-}$, the convergence of the IBVP solutions $(v, u)(x, t)$ to the constant solutions $(\bar{v}, \bar{u})(x, t)=\left(v_{+}, 0\right)$ will be discussed in the last part of this paper. Therein, instead of the boundary condition (1.7), we will consider a boundary condition on $u$.

Now, let us assume for the moment that $v_{+} \neq v_{-}$. In general, we will consider the situation in which $g(t)$ converges as $t \rightarrow+\infty$. As a prototype of this situation we will investigate the case where $g(t) \rightarrow v_{+}$as $t \rightarrow+\infty$. To overcome the difficulty of large boundary perturbations,

$$
\left.v\right|_{x=0}-\left.\bar{v}(x / \sqrt{t+1})\right|_{x=0}=g(t)-\bar{v}(0) \rightarrow v_{+}-\bar{v}(0) \neq 0 \quad \text { as } t \rightarrow+\infty,
$$

we will introduce a suitable time-dependent shift function on the time $t$. Such a technique was used to treat the convergence to travelling waves in $[12,11,17,18]$ for some examples of conservation laws with the boundary conditions. 
To be consistent with the known decay estimates for the corresponding Cauchy problem given in $[7,19]$, we assume that

$$
\left|g(t)-v_{+}\right|=O(1)\left|v_{+}-v_{-}\right|(1+t)^{-\gamma_{1}}, \quad \gamma_{1}>3 / 4
$$

and the compatibility condition

$$
g(0)=v_{0}(0)
$$

Since the nonlinear diffusion wave $\bar{v}(x, t)=\phi(x / \sqrt{t+1})$ of $(1.2)$ satisfies (see $[7,19])$

$$
\left|v_{+}-\bar{v}(x, t)\right| \leq C\left|v_{+}-v_{-}\right| e^{-c_{0} \alpha \xi^{2}}, \quad \xi=x / \sqrt{t+1}, \quad \text { for } x \geq 0
$$

for some constants $C>0$ and $c_{0}>0$, let us choose the shift function $d(t)$ in $C^{3}\left(\mathbb{R}_{+}\right)$ such that

$$
\begin{gathered}
d(t)>0 \quad \text { for all } t \geq 0, \\
\exp \left\{-\alpha c_{0}\left(\frac{d(t)}{\sqrt{t+1}}\right)^{2}\right\} \leq O(1)(2+t)^{-\gamma_{2}}, \quad \gamma_{2}>3 / 4, \\
d^{\prime}(t) \exp \left\{-\alpha c_{0}\left(\frac{d(t)}{\sqrt{t+1}}\right)^{2}\right\} \leq O(1)(1+t)^{-\left(\gamma_{2}+\frac{1}{2}\right)} \sqrt{\log (2+t)} .
\end{gathered}
$$

Here, we denote $d(0)=d_{0}$. The function $d(t)$ satisfying (1.11)-(1.13) includes many examples. Two kinds of important examples are $d(t)=\sqrt{1+t} \cdot \sqrt{c \log (2+t)}$ with $c \geq \gamma_{2} /\left(\alpha c_{0}\right)$ and $d(t)=(1+t)^{\frac{1}{2}+c}$ with any $c>0$. Note that the choice $d(t)=$ $\sqrt{1+t} \cdot \sqrt{c_{1} \log (2+t)}$ with $c_{1}=\gamma_{2} /\left(\alpha c_{0}\right)$ is the weakest one in the sense of optimal decay rates.

Because of the second equation of (1.1), we have

$$
u(x, t) \rightarrow e^{-\alpha t} u_{+} \quad \text { as } x \rightarrow+\infty
$$

and the implicit relation

$$
\left.u\right|_{x=0}=e^{-\alpha t} u_{0}(0)-\int_{0}^{t} e^{-\alpha(t-\tau)} p^{\prime}(g(\tau)) v_{x}(0, \tau) d \tau .
$$

Let us denote $(\bar{v}, \bar{u})=(\bar{v}, \bar{u})(x+d(t), t)$ : the shifted nonlinear diffusion waves of (1.2). By (1.3) we get

$$
\frac{d}{d t} \bar{v}(x+d(t), t)=d^{\prime}(t) \bar{v}_{x}(x+d(t), t)+\bar{u}_{x}(x+d(t), t)
$$

and by (1.4) and (1.5) we have

$$
(\bar{v}, \bar{u}) \rightarrow\left(v_{+}, 0\right) \quad \text { as } x \rightarrow+\infty .
$$

Denote

$$
\left\{\begin{array}{l}
\hat{v}(x, t):=-\frac{u_{+}-u_{0}(0)}{\alpha} e^{-\alpha t} m_{0}(x), \\
\hat{u}(x, t):=e^{-\alpha t}\left[u_{+}-\left(u_{+}-u_{0}(0)\right) \int_{x}^{\infty} m_{0}(y) d y\right],
\end{array}\right.
$$

where $m_{0}(x)$ is a $C_{0}^{\infty}\left(\mathbb{R}_{+}\right)$function satisfying

$$
m_{0} \geq 0 \quad \text { for all } x \in \mathbb{R}_{+}, \quad m_{0}(0)=0 \quad \text { and } \quad \int_{0}^{+\infty} m_{0}(x) d x=1 .
$$


We can easily check that

$$
\left\{\begin{array}{l}
\hat{v}_{t}-\hat{u}_{x}=0, \\
\hat{u}_{t}=-\alpha \hat{u},
\end{array}\right.
$$

and the following boundary and limit conditions hold:

$$
\begin{gathered}
\left.(\hat{v}, \hat{u})\right|_{x=+\infty}=\left(0, e^{-\alpha t} u_{+}\right), \\
\left.(\hat{v}, \hat{u})\right|_{x=0}=\left(0, u_{0}(0) e^{-\alpha t}\right), \\
|\hat{v}| \leq \alpha^{-1}\left|u_{+}-u_{0}(0)\right| e^{-\alpha t} m_{0}(x) .
\end{gathered}
$$

From (1.1), (1.16), and (1.20), we have

$$
(v-\bar{v}-\hat{v})_{t}=\left(u-\bar{u}-\hat{u}-d^{\prime}(t) \bar{v}\right)_{x} ;
$$

by integrating it over $[x,+\infty)$ and by using that $\left.(v-\bar{v}-\hat{v})\right|_{x=+\infty}=0,\left.(u-\bar{u}-\hat{u})\right|_{x=+\infty}=0$ and $(1.15),(1.22)$ we have

$$
\begin{aligned}
& \frac{d}{d t} \int_{0}^{\infty}(v(x, t)-\bar{v}(x+d(t), t)-\hat{v}(x, t)) d x \\
& \quad=-\left.(u-\bar{u}-\hat{u})\right|_{x=0}-d^{\prime}(t)\left[v_{+}-\bar{v}(d(t), t)\right] \\
& \quad=\int_{0}^{t} e^{-\alpha(t-\tau)} p^{\prime}(g(\tau)) v_{x}(0, \tau) d \tau+\bar{u}(d(t), t)-d^{\prime}(t)\left[v_{+}-\bar{v}(d(t), t)\right] .
\end{aligned}
$$

Since $v_{x}(0, t)$ can be controlled automatically by the equations (1.1), we conjecture that the right-hand side of (1.25) is integrable and the integration tends to zero as $t$ goes to infinity, namely,

$$
\begin{aligned}
& \text { (Ansatz) }: \mid \int_{0}^{\infty}(v(x, t)-\bar{v}(x+d(t), t)-\hat{v}(x, t)) d x \mid \\
&= \mid \int_{0}^{\infty}\left(v_{0}(x)-\bar{v}\left(x+d_{0}, 0\right)-\hat{v}(x, 0)\right) d x \\
&+\int_{0}^{t}\left\{\int_{0}^{\tau} e^{-\alpha(\tau-\eta)} p^{\prime}(g(\eta)) v_{x}(0, \eta) d \eta\right. \\
&\left.\quad+\bar{u}(d(\tau), \tau)-d^{\prime}(\tau)\left[v_{+}-\bar{v}(d(\tau), \tau)\right] d \tau\right\} \mid \\
& \leq O(1)(1+t)^{-1 / 4} \text { as } t \rightarrow+\infty .
\end{aligned}
$$

In the next sections, we will prove that this ansatz is true.

Due to the previous analysis, let us define the new variables

$$
\begin{aligned}
V(x, t) & :=-\int_{x}^{+\infty}[v(y, t)-\bar{v}(y+d(t), t)-\hat{v}(y, t)] d y, \\
z(x, t) & :=u(x, t)-\bar{u}(x+d(t), t)-\hat{u}(x, t) .
\end{aligned}
$$

Thus, the ansatz (1.26) is equivalent to showing that

$$
(\text { Ansatz })^{\prime}:|V|_{x=0} \mid \leq O(1)(1+t)^{-1 / 4} \text { as } t \rightarrow+\infty \text {. }
$$

It will be answered below; see Theorem 2.1, Corollary 2.2, and Remark 2.3 in Sec. 2. 
From the equations $(1.1),(1.3),(1.16),(1.20)$, and $(1.27)$, we can reformulate the original equations (1.1) to the new one

$$
\left\{\begin{array}{l}
V_{x t}=z_{x}-d^{\prime}(t) \bar{v}_{x}, \\
z_{t}+\alpha z+\left(p^{\prime}(\bar{v}) V_{x}\right)_{x}=f_{1},
\end{array} \quad(x, t) \in \mathbb{R}_{+} \times \mathbb{R}_{+}\right.
$$

where

$$
f_{1}:=-\frac{d}{d t} \bar{u}(x+d(t), t)-\left\{p\left(V_{x}+\bar{v}+\hat{v}\right)-p(\bar{v})-p^{\prime}(\bar{v}) V_{x}\right\}_{x} .
$$

Since $\left.V\right|_{x=+\infty}=\left.z\right|_{x=+\infty}=0,\left.\bar{v}\right|_{x=+\infty}=v_{+}$, then by integrating the first equation of $(1.29)$ over $[x,+\infty)$, one has

$$
\left\{\begin{array}{l}
V_{t}=z+d^{\prime}(t)\left[v_{+}-\bar{v}\right], \\
z_{t}+\alpha z+\left(p^{\prime}(\bar{v}) V_{x}\right)_{x}=f_{1},
\end{array} \quad(x, t) \in \mathbb{R}_{+} \times \mathbb{R}_{+} .\right.
$$

The corresponding initial data are given by

$$
\left.\left(V, V_{t}, z\right)\right|_{t=0}=\left(V_{0}, V_{1}, z_{0}\right)(x), \quad x \in \mathbb{R}_{+},
$$

where

$$
\begin{aligned}
V_{0}(x) & :=-\int_{x}^{+\infty}\left[v_{0}(y)-\bar{v}\left(y+d_{0}, 0\right)-\hat{v}(y, 0)\right] d y \\
z_{0}(x) & :=u_{0}(x)-\bar{u}\left(x+d_{0}, 0\right)-\hat{u}(x, 0) \\
V_{1}(x) & :=z_{0}(x)+d^{\prime}(0)\left[v_{+}-\bar{v}\left(x+d_{0}, 0\right)\right]
\end{aligned}
$$

and the boundary value is given by (1.7) and (1.22):

$$
\begin{aligned}
\left.V_{x}\right|_{x=0} & =\left.(v-\bar{v}-\hat{v})\right|_{x=0}=g(t)-\bar{v}(d(t), t) \\
& =\left[g(t)-v_{+}\right]+\left[v_{+}-\bar{v}(d(t), t)\right]=: G(t), \quad t \in \mathbb{R}_{+} .
\end{aligned}
$$

Plugging the first equation of (1.31) into the second equation of (1.31), we have the following Neumann type IBVP:

$$
\left\{\begin{array}{l}
L(V):=V_{t t}+\alpha V_{t}+\left(p^{\prime}(\bar{v}) V_{x}\right)_{x}=F, \quad x>0, t>0 \\
\left.\left(V, V_{t}\right)\right|_{t=0}=\left(V_{0}, V_{1}\right)(x), \quad x>0 \\
\left.V_{x}\right|_{x=0}=G(t), \quad t>0
\end{array}\right.
$$

where $F:=f_{1}+f_{2}$ and

$$
f_{2}:=d^{\prime \prime}(t)\left(v_{+}-\bar{v}\right)-d^{\prime}(t) \frac{d}{d t} \bar{v}(x+d(t), t)+\alpha d^{\prime}(t)\left(v_{+}-\bar{v}\right)
$$

From the compatibility condition (1.9), we may easily check the other compatibility condition $G(0)=V_{0, x}(0)$ for the IBVP (1.37).

In the following two sections, we will prove that the IBVP (1.37) has a unique global solution with some algebraic decay rates in the $L^{2}$-sense by the elementary energy method.

Notation. Here and after here, we denote several generic constants by $c$ or $C$, or $c_{i}, C_{i}$. $H^{k}\left(\mathbb{R}_{+}\right)$is the usual Sobolev space with the norm

$$
\|f\|_{k}=\sum_{i=0}^{k}\left\|\partial_{x}^{i} f\right\|
$$


where $\|f\|=\left(\int_{0}^{+\infty} f(x)^{2} d x\right)^{1 / 2}$ is the norm of $L^{2}\left(\mathbb{R}_{+}\right) . W^{k, \infty}\left(0, T ; H^{l}\right)(k \geq 0, l \geq$ $0,0<T \leq+\infty)$ is the space of $H^{l}$-valued $k$-times differentiable functions on $[0, T]$.

2. Nonlinear diffusion waves and main theorem. In this section, we firstly recall the properties of the nonlinear diffusion waves. Then we are going to state our main result on the convergence to the suitable diffusion waves.

As shown in [6], [7], and [19], since the nonlinear diffusion equation

$$
\tau_{t}=-\frac{1}{\alpha} p(\tau)_{x x}, \quad p^{\prime}(\tau)<0,
$$

is invariant under the transformation $(x, t) \rightarrow\left(c x, c^{2} t\right), c>0$, then it has self-similar solutions called "nonlinear diffusion waves", namely solutions in the form

$$
\tau^{*}(x, t)=\phi(x / \sqrt{t}):=\phi(\xi), \quad \xi \in \mathbb{R},
$$

with $\phi( \pm \infty)=v_{ \pm}$. The function $\phi$ satisfies

$$
\sum_{k=1}^{3}\left|\frac{d^{k}}{d \xi^{k}} \phi(\xi)\right|+\left|\phi(\xi)-v_{+}\right|_{\xi>0}+\left|\phi(\xi)-v_{-}\right|_{\xi<0} \leq C\left|v_{+}-v_{-}\right| e^{-c \alpha \xi^{2}}
$$

and hence $\tau^{*}(x, t)$ satisfies

$$
\begin{aligned}
& \tau_{x}^{*}=\frac{\phi^{\prime}(\xi)}{\sqrt{t}}, \quad \tau_{t}^{*}=-\frac{\xi \phi^{\prime}(\xi)}{2 t}, \quad \tau_{x x}^{*}=\frac{\phi^{\prime \prime}(\xi)}{t}, \quad \tau_{x t}^{*}=\frac{\phi^{\prime}(\xi)+\xi \phi^{\prime \prime}(\xi)}{2 t \sqrt{t}} \\
& \tau_{t t}^{*}=\frac{3 \xi \phi^{\prime}(\xi)+\xi^{2} \phi^{\prime \prime}(\xi)}{4 t^{2}}, \quad \tau_{x x x}^{*}=\frac{\phi^{\prime \prime \prime}(\xi)}{t \sqrt{t}}, \quad \tau_{x t t}^{*}=\frac{3 \phi^{\prime}(\xi)+5 \xi \phi^{\prime \prime}(\xi)+\xi^{2} \phi^{\prime \prime \prime}(\xi)}{4 t^{2} \sqrt{t}},
\end{aligned}
$$

and the following decay estimates:

$$
\begin{aligned}
\int_{-\infty}^{\infty}\left|\tau_{x}^{*}(x, t)\right|^{2} d t & =O(1)\left|v_{+}-v_{-}\right|^{2} t^{-1 / 2}, \\
\int_{-\infty}^{\infty}\left(\left|\tau_{t}^{*}\right|^{2}+\left|\tau_{x x}^{*}\right|^{2}\right) d t & =O(1)\left|v_{+}-v_{-}\right|^{2} t^{-3 / 2}, \\
\int_{-\infty}^{\infty}\left(\left|\tau_{x t}^{*}\right|^{2}+\left|\tau_{x x x}^{*}\right|^{2}\right) d t & =O(1)\left|v_{+}-v_{-}\right|^{2} t^{-5 / 2}, \\
\int_{-\infty}^{\infty}\left|\tau_{t t}^{*}(x, t)\right|^{2} d t & =O(1)\left|v_{+}-v_{-}\right|^{2} t^{-7 / 2}, \\
\int_{-\infty}^{\infty}\left|\tau_{x t t}^{*}(x, t)\right|^{2} d t & =O(1)\left|v_{+}-v_{-}\right|^{2} t^{-9 / 2} .
\end{aligned}
$$

To avoid the singularity at $t=0$, we prefer to set

$$
\bar{v}(x, t):=\tau^{*}(x, t+1)=\phi(x / \sqrt{1+t}) .
$$

Our main theorem can be stated as follows.

Theorem 2.1. Suppose that $v_{0} \in H^{3}\left(\mathbb{R}_{+}\right), v_{1} \in H^{2}\left(\mathbb{R}_{+}\right)$and denote by $\delta=\left|v_{+}-v_{-}\right|+$ $\left|u_{+}-u_{0}(0)\right|$. Then there exist a constant $\varepsilon_{1}>0$ such that if $\left\|V_{0}\right\|_{3}+\left\|V_{1}\right\|_{2}+\delta \leq \varepsilon_{1}$, 
then the IBVP (1.37) has a unique globally defined solution $V(x, t)$ satisfying

$$
V \in \bigcap_{i=0}^{3} W^{i, \infty}\left([0, \infty) ; H^{3-i}\right)
$$

and

$$
\begin{aligned}
& \sum_{i=0}^{3}(1+t)^{i}\left\|\partial_{x}^{i} V(\cdot, t)\right\|^{2}+\sum_{i=0}^{2}(1+t)^{i+2}\left\|\partial_{x}^{i} V_{t}(\cdot, t)\right\|^{2} \\
& \quad+\int_{0}^{t}\left[\sum_{i=1}^{3}(1+\tau)^{i-1}\left\|\partial_{x}^{i} V(\cdot, \tau)\right\|^{2}+\sum_{i=0}^{2}(1+\tau)^{i+1}\left\|\partial_{x}^{i} V_{t}(\cdot, \tau)\right\|^{2}\right] d \tau \\
& \quad \leq C\left(\left\|V_{0}\right\|_{3}^{2}+\left\|V_{1}\right\|_{2}^{2}+\delta\right) .
\end{aligned}
$$

By using the inequality $\|f\|_{L^{\infty}} \leq \sqrt{2}\|f\|^{1 / 2}\left\|f_{x}\right\|^{1 / 2}$, Theorem 2.1 yields to the following sup-norm estimates.

Corollary 2.2. Under the previous hypotheses, one has

$$
\begin{aligned}
\|V(\cdot, t)\|_{L^{\infty}} & \leq C(1+t)^{-1 / 4}, \\
\left\|V_{x}(\cdot, t)\right\|_{L^{\infty}} & \leq C(1+t)^{-3 / 4} \\
\left\|V_{t}(\cdot, t)\right\|_{L^{\infty}} & \leq C(1+t)^{-5 / 4} \\
\left\|V_{x x}(\cdot, t)\right\|_{L^{\infty}} & \leq C(1+t)^{-5 / 4} \\
\left\|V_{x t}(\cdot, t)\right\|_{L^{\infty}} & \leq C(1+t)^{-7 / 4} .
\end{aligned}
$$

REMARK 2.3. From (2.9), we see that our Ansatz (1.28) or (1.26) is true, namely $|V(0, t)| \leq\|V(\cdot, t)\|_{L^{\infty}} \leq C(1+t)^{-1 / 4}$.

3. A priori estimates. Since we intend to apply the classical continuation method, we will give a proof of Theorem 2.1 based on a local existence result together with higherorder a priori estimates, which are the core argument. Since the local existence can be proved by a standard method, see, for instance, Matsumura [16] and Nishida [20], our main effort, in this section, will be to prove the a priori estimates. The outline of the proof is quite similar to that one in the paper of Nishihara [19].

Let us define

$$
N(T)^{2}:=\sup _{0 \leq t \leq T}\left\{\sum_{i=0}^{3}(1+t)^{i}\left\|\partial_{x}^{i} V(\cdot, t)\right\|^{2}+\sum_{i=0}^{2}(1+t)^{i+2}\left\|\partial_{x}^{i} V_{t}(\cdot, t)\right\|^{2}\right\}
$$

for any $T \in[0,+\infty]$. We will prove our estimates in five steps. Our estimates will provide both the a priori bounds and the decay rate at the same moment.

Step 1. The decay rate for $V_{x}$ and $V_{t}$. We begin with the first-order energy estimate.

LEMMA 3.1. It follows that

$$
\left\|\left(V, V_{x}, V_{t}\right)(t)\right\|^{2}+\int_{0}^{t}\left\|\left(V_{x}, V_{t}\right)(\tau)\right\|^{2} d \tau \leq C\left(\left\|\left(V_{0}, V_{0, x}, V_{1}\right)\right\|^{2}+\delta\right)
$$

provided $N(T)+\delta \ll 1$. 
Proof. By multiplying (1.37) by $\lambda V+V_{t}(0<\lambda \ll 1)$, it follows that

$$
\left\{E_{1}\left(V, V_{t}, V_{x}\right)\right\}_{t}+E_{2}\left(V_{x}, V_{t}\right)+\left\{B_{1}(x, t)\right\}_{x}=F \cdot\left(\lambda V+V_{t}\right),
$$

where

$$
\begin{aligned}
E_{1}\left(V, V_{x}, V_{t}\right) & :=\frac{1}{2} V_{t}^{2}+\lambda V V_{t}+\frac{\lambda \alpha}{2} V^{2}-\frac{1}{2} p^{\prime}(\bar{v}) V_{x}^{2}, \\
E_{2}\left(V_{x}, V_{t}\right) & :=(\alpha-\lambda) V_{t}^{2}+\left(-\lambda p^{\prime}(\bar{v})+\frac{1}{2} p^{\prime \prime}(\bar{v})\left(\bar{v}_{t}+d^{\prime}(t) \bar{v}_{x}\right)\right) V_{x}^{2}, \\
B_{1}(x, t) & :=p^{\prime}(\bar{v}) V_{x}\left(\lambda V+V_{t}\right) .
\end{aligned}
$$

It is clear that, when $0<\lambda \ll 1$, we have constants $C_{1}>0$ and $C_{1}^{\prime}>0$, such that

$$
C_{1}\left(V^{2}+V_{x}^{2}+V_{t}^{2}\right) \leq E_{1}\left(V, V_{x}, V_{t}\right) \leq C_{1}^{\prime}\left(V^{2}+V_{x}^{2}+V_{t}^{2}\right) .
$$

Since $-p^{\prime}(\bar{v})>0,\left|p^{\prime \prime}(\bar{v})\right| \leq C, 0<d^{\prime}(t)=O(1)(1+t)^{-1 / 2}(\log (2+t))^{1 / 2} \leq C$, and $\left|\bar{v}_{t}\right| \leq C\left|v_{+}-v_{-}\right|,\left|\bar{v}_{x}\right| \leq C \mid v_{+}-v_{-}$, letting $\left|v_{+}-v_{-}\right| \leq \delta \ll \lambda$, we obtain for some $C_{2}>0$

$$
E_{2}\left(V_{x}, V_{t}\right) \geq C_{2}\left(V_{x}^{2}+V_{t}^{2}\right) .
$$

Now we deduce the boundary estimate. By using (1.8), (2.3), and (1.12), one has

$$
\begin{aligned}
|G(t)| & =\left|\left[g(t)-v_{+}\right]+\left[v_{+}-\bar{v}(d(t), t)\right]\right| \\
& \leq O(1) \delta\left[(1+t)^{-\gamma_{1}}+\exp \left(-\alpha c_{0}\left(\frac{d(t)}{\sqrt{1+t}}\right)^{2}\right)\right] \\
& \leq O(1) \delta\left[(1+t)^{-\gamma_{1}}+(1+t)^{-\gamma_{2}}\right] \\
& \leq O(1) \delta(1+t)^{-\gamma_{3}}
\end{aligned}
$$

where $\gamma_{3}:=\min \left\{\gamma_{1}, \gamma_{2}\right\}>\frac{3}{4}\left(\right.$ since $\left.\gamma_{1}, \gamma_{2}>\frac{3}{4}\right)$. Therefore, it follows that

$$
\begin{aligned}
& (1+t)^{1 / 4} \sup _{x \in R_{+}}|V(x, t)|+(1+t)^{5 / 4} \sup _{x \in R_{+}}\left|V_{t}(x, t)\right| \\
& \leq \sqrt{2}(1+t)^{1 / 4}\|V(t)\|^{1 / 2}\left\|V_{x}(t)\right\|^{1 / 2}+\sqrt{2}(1+t)^{5 / 4}\left\|V_{x}(t)\right\|^{1 / 2}\left\|V_{t}(t)\right\|^{1 / 2} \\
& \quad \leq C N(t) .
\end{aligned}
$$

Then the boundary integration can be controlled as follows. Since $\gamma_{3}>3 / 4$,

$$
\begin{aligned}
\left|\int_{0}^{t} B_{1}(0, \tau) d \tau\right| & =\left|\int_{0}^{t} p^{\prime}\left(\left.\bar{v}\right|_{x=0}\right) G(\tau)\left[V_{t}(0, \tau)+\lambda V_{x}(0, \tau)\right] d \tau\right| \\
& \leq C \delta N(t) \int_{0}^{t}(1+\tau)^{-\gamma_{3}}\left[(1+\tau)^{-5 / 4}+(1+\tau)^{-1 / 4}\right] d \tau \\
& \leq C \delta N(t) .
\end{aligned}
$$

Integrating (3.3) over $\mathbb{R}_{+} \times[0, t]$ and using (3.7), (3.8), and (3.11), we get

$$
\begin{aligned}
\left\|\left(V, V_{x}, V_{t}\right)(t)\right\|^{2}+\int_{0}^{t} & \left\|\left(V_{x}, V_{t}\right)(\tau)\right\|^{2} d \tau \\
& \leq C\left(\left\|\left(V_{0}, V_{0, x}, V_{1}\right)\right\|^{2}+\delta\right)+C \int_{0}^{t} \int_{0}^{\infty} F \cdot\left(\lambda V+V_{t}\right) d x d \tau .
\end{aligned}
$$


Similar to (3.11), by Taylor's formulas,

$$
\left|p\left(V_{x}+\bar{v}+\hat{v}\right)-p\left(V_{x}+\bar{v}\right)\right|=O(1)|\hat{v}|
$$

and

$$
\left|p\left(V_{x}+\bar{v}\right)-p(\bar{v})-p^{\prime}(\bar{v}) V_{x}\right|=O(1)\left|V_{x}\right|^{2}
$$

and by $\left.\bar{v}\right|_{x=0}=0$ (see (1.19)), $\left.V_{x}\right|_{x=0}=G(t)$, we have another boundary decay as follows:

$$
\begin{aligned}
&\left|\left[\left(p\left(V_{x}+\bar{v}+\hat{v}\right)-p(\bar{v})-p^{\prime}(\bar{v}) V_{x}\right)\left(\lambda V+V_{t}\right)\right]\right|_{x=0} \mid \\
& \leq\left|\left[\left(p\left(V_{x}+\bar{v}+\hat{v}\right)-p\left(V_{x}+\bar{v}\right)\right)\left(\lambda V+V_{t}\right)\right]\right|_{x=0} \mid \\
&+\left|\left[\left(p\left(V_{x}+\bar{v}\right)-p(\bar{v})-p^{\prime}(\bar{v}) V_{x}\right)\left(\lambda V+V_{t}\right)\right]\right|_{x=0} \mid \\
& \leq\left.C\left|\left[\hat{v}\left(\lambda V+V_{t}\right)\right]\right|_{x=0}|+C|\left[V_{x}^{2}\left(\lambda V+V_{t}\right)\right]\right|_{x=0} \mid \\
& \leq C \delta N(t) G(t)^{2}\left[\lambda(1+t)^{-1 / 4}+(1+t)^{-5 / 4}\right] \\
& \leq C \delta N(t)\left[(1+t)^{-\left(2 \gamma_{1}+\frac{1}{4}\right)}+(1+t)^{-\left(2 \gamma_{1}+\frac{5}{4}\right)}\right] \\
& \leq C \delta N(t)(1+t)^{-\left(2 \gamma_{1}+\frac{1}{4}\right)} .
\end{aligned}
$$

Now, we are going to estimate the integration dealing with the first part $f_{1}$ of the nonlinear term $F$. First by (1.30) and by integrating by parts with respect to $x$, we can rewrite it as follows:

$$
\begin{aligned}
\int_{0}^{t} \int_{0}^{\infty} f_{1} \cdot\left(\lambda V+V_{t}\right) d x d \tau \\
=\int_{0}^{t} \int_{0}^{\infty}-\frac{d}{d t} \bar{u}(x+d(\tau), \tau)\left(\lambda V+V_{t}\right) d x d \tau \\
\quad+\left.\int_{0}^{t}\left[\left(p\left(V_{x}+\bar{v}+\hat{v}\right)-p(\bar{v})-p^{\prime}(\bar{v}) V_{x}\right)\left(\lambda V+V_{t}\right)\right]\right|_{x=0} d \tau \\
\quad+\int_{0}^{t} \int_{0}^{\infty}\left(p\left(V_{x}+\bar{v}+\hat{v}\right)-p(\bar{v})-p^{\prime}(\bar{v}) V_{x}\right)\left(\lambda V_{x}+V_{x t}\right) d x d \tau \\
=: I_{1}+I_{2}+I_{3} .
\end{aligned}
$$

From (1.2), (2.3), and (2.4), we note

$$
\begin{aligned}
\left|\bar{u}_{x}\right| & =\left|p^{\prime}(\bar{v}) \bar{v}_{x x}+p^{\prime \prime}(\bar{v}) \bar{v}_{x}^{2}\right| \\
& \leq C\left|v_{+}-v_{-}\right|(1+t)^{-1} \exp \left(-c_{0} \alpha\left(\frac{x+d(t)}{\sqrt{t+1}}\right)^{2}\right), \\
\left|\bar{u}_{t}\right| & =\left|p^{\prime}(\bar{v}) \bar{v}_{x t}+p^{\prime \prime}(\bar{v}) \bar{v}_{x} \bar{v}_{t}\right| \\
& \leq C\left|v_{+}-v_{-}\right|(1+t)^{-3 / 2} \exp \left(-c_{0} \alpha\left(\frac{x+d(t)}{\sqrt{t+1}}\right)^{2}\right) .
\end{aligned}
$$


Then, due to (3.17), (3.18), (1.12), (1.13), and the inequality $(3.10)$, since $d(t)>0, I_{1}$ can be controlled in the following way:

$$
\begin{aligned}
I_{1}= & \int_{0}^{t} \int_{0}^{\infty}-\left(d^{\prime}(\tau) \bar{u}_{x}+\bar{u}_{t}\right)\left(\lambda V+V_{t}\right) d x d \tau \\
\leq & C\left|v_{+}-v_{-}\right| N(t) \int_{0}^{t} \int_{0}^{\infty}\left[d^{\prime}(\tau)(1+\tau)^{-1}+(1+\tau)^{-3 / 2}\right] \\
& \cdot \exp \left(-c_{0} \alpha\left(\frac{x+d(\tau)}{\sqrt{\tau+1}}\right)^{2}\right)\left[\lambda(1+\tau)^{-1 / 4}+(1+\tau)^{-5 / 4}\right] d x d \tau \\
\leq & C\left|v_{+}-v_{-}\right| N(t) \int_{0}^{t}\left[(1+\tau)^{-3 / 2}(\log (2+\tau))^{1 / 2}+(1+\tau)^{-3 / 2}\right] \\
& \cdot\left[\lambda(1+\tau)^{-1 / 4}+(1+\tau)^{-5 / 4}\right](1+\tau)^{1 / 2} \exp \left(-c_{0} \alpha\left(\frac{d(\tau)}{\sqrt{\tau+1}}\right)^{2}\right) d \tau \\
& \cdot \int_{0}^{\infty} \exp \left(-c_{0} \alpha\left(\frac{x}{\sqrt{\tau+1}}\right)^{2}\right) d \frac{x}{\sqrt{\tau+1}} \\
\leq & C\left|v_{+}-v_{-}\right| N(t) \int_{0}^{t}(1+\tau)^{-\left(\gamma_{2}+\frac{\tau}{4}\right)}\left[1+(\log (2+\tau))^{1 / 2}\right] d \tau \\
\leq & C \delta N(t) .
\end{aligned}
$$

On the other hand, the boundary integration $I_{2}$ can easily be controlled from (3.15) and $\gamma_{1}>3 / 4$. Then

$$
I_{2} \leq C \delta N(t) \int_{0}^{t}(1+\tau)^{-\left(2 \gamma_{1}+\frac{1}{4}\right)} \leq C \delta N(t) .
$$

Finally, we are going to estimate $I_{3}$. By the Sobolev inequality, $(1+t)^{3 / 4} \sup _{x \in R_{+}}\left|V_{x}(x, t)\right|$ $\leq C N(t)$ and since $|\hat{v}| \leq C \delta e^{-\alpha t} m_{0}(x), m_{0}(x) \geq 0$, see (1.23), we have

$$
\begin{aligned}
\int_{0}^{t} \int_{0}^{\infty}\left(p\left(V_{x}+\bar{v}+\hat{v}\right)-p(\bar{v})-p^{\prime}(\bar{v}) V_{x}\right) \lambda V_{x} d x d \tau \\
\leq \int_{0}^{t} \int_{0}^{\infty}\left|\left(p\left(V_{x}+\bar{v}+\hat{v}\right)-p\left(V_{x}+\bar{v}\right)\right) \lambda V_{x}\right| d x d \tau \\
\quad+C \int_{0}^{t} \int_{0}^{\infty}\left|\left(p\left(V_{x}+\bar{v}\right)-p(\bar{v})-p^{\prime}(\bar{v}) V_{x}\right) \lambda V_{x}\right| d x d \tau \\
\leq C \int_{0}^{t} \int_{0}^{\infty}\left|\hat{v} V_{x}\right| d x d \tau+C \int_{0}^{t} \int_{0}^{\infty}\left|V_{x}^{3}\right| d x d \tau \\
\leq C \delta N(t) \int_{0}^{t} \int_{0}^{\infty} e^{-\alpha \tau}(1+\tau)^{-3 / 4} m_{0}(x) d x d \tau \\
\quad+C N(t) \int_{0}^{t} \int_{0}^{\infty}(1+\tau)^{-3 / 4} V_{x}^{2} d x d \tau \\
\leq C \delta N(t)+C N(t) \int_{0}^{t}\left\|V_{x}(\tau)\right\|^{2} d \tau .
\end{aligned}
$$


Now let us observe that

$$
\begin{aligned}
\left(p \left(V_{x}+\right.\right. & \left.\bar{v}+\hat{v})-p(\bar{v})-p^{\prime}(\bar{v}) V_{x}\right) V_{x t} \\
= & \frac{d}{d t}\left\{\int_{\bar{v}}^{V_{x}+\bar{v}+\hat{v}} p(s) d s-p(\bar{v}) V_{x}-\frac{1}{2} p^{\prime}(\bar{v}) V_{x}^{2}\right\} \\
& -\left[p\left(V_{x}+\bar{v}+\hat{v}\right)-p(\bar{v})-p^{\prime}(\bar{v}) V_{x}\right] \frac{d}{d t} \bar{v} \\
& -p\left(V_{x}+\bar{v}+\hat{v}\right) \hat{v}_{t}+\frac{1}{2} p^{\prime \prime}(\bar{v}) \frac{d \bar{v}}{d t} V_{x}^{2}
\end{aligned}
$$

Therefore, denoting by

$$
H(y):=\int_{\bar{v}}^{y} p(s) d s \quad \text { for } y \in \mathbb{R}
$$

one has

$$
H\left(V_{x}+\bar{v}+\hat{v}\right)=\int_{\bar{v}}^{V_{x}+\bar{v}+\hat{v}} p(s) d s, \quad H(\bar{v})=0, \quad H^{\prime}(\bar{v})=p(\bar{v}), \quad \text { and } \quad H^{\prime \prime}(\bar{v})=p^{\prime}(\bar{v}) .
$$

Thus, Taylor's formula

$$
H\left(V_{x}+\bar{v}+\hat{v}\right)=H(\bar{v})+H^{\prime}(\bar{v})\left(V_{x}+\hat{v}\right)+\frac{1}{2} H^{\prime \prime}(\bar{v})\left(V_{x}+\hat{v}\right)^{2}+O(1)\left(V_{x}+\hat{v}\right)^{3}
$$

leads to the following identity:

$$
\int_{\bar{v}}^{V_{x}+\bar{v}+\hat{v}} p(s) d s=p(\bar{v})\left(V_{x}+\hat{v}\right)+\frac{1}{2} p^{\prime \prime}(\bar{v})\left(V_{x}+\hat{v}\right)^{2}+O(1)\left(V_{x}+\hat{v}\right)^{3}
$$

namely,

$$
\begin{gathered}
\int_{\bar{v}}^{V_{x}+\bar{v}+\hat{v}} p(s) d s-p(\bar{v}) V_{x}-\frac{1}{2} p^{\prime}(\bar{v}) V_{x}^{2} \\
=p(\bar{v}) \hat{v}+\frac{1}{2} p^{\prime \prime}(\bar{v})\left(2 V_{x} \hat{v}+\hat{v}^{2}\right)+O(1)\left(V_{x}+\hat{v}\right)^{3}
\end{gathered}
$$

By means of the same calculation as used in (3.21), we can prove

$$
\begin{gathered}
\int_{0}^{\infty}\left[p(\bar{v}) \hat{v}+\frac{1}{2} p^{\prime \prime}(\bar{v})\left(2 V_{x} \hat{v}+\hat{v}^{2}\right)+O(1)\left(V_{x}+\hat{v}\right)^{3}\right] d x \\
\leq C \delta(1+N(t))+C N(t)\left\|V_{x}(t)\right\|^{2}
\end{gathered}
$$


so that from (3.23), we get

$$
\begin{aligned}
& \int_{0}^{\infty}\left\{\int_{\bar{v}}^{V_{x}+\bar{v}+\hat{v}} p(s) d s-p(\bar{v}) V_{x}-\frac{1}{2} p^{\prime}(\bar{v}) V_{x}^{2}\right\} d x \\
& \leq C \delta(1+N(t))+C N(t)\left\|V_{x}(t)\right\|^{2} .
\end{aligned}
$$

Similarly, thanks to (1.11)-(1.13), (1.18), (1.19), (1.23), and (2.3)-(2.5), by using the Taylor expansions (3.13) and (3.14), we show

$$
\begin{aligned}
\int_{0}^{t} \int_{0}^{\infty}-\left[p\left(V_{x}+\bar{v}+\hat{v}\right)-p(\bar{v})-p^{\prime}(\bar{v}) V_{x}\right] \frac{d}{d t} \bar{v} d x d \tau \\
=\int_{0}^{t} \int_{0}^{\infty}-\left[p\left(V_{x}+\bar{v}+\hat{v}\right)-p\left(V_{x}+\bar{v}\right)\right]\left[d^{\prime}(\tau) \bar{v}_{x}+\bar{v}_{t}\right] d x d \tau \\
\quad-\int_{0}^{t} \int_{0}^{\infty}\left[p\left(V_{x}+\bar{v}\right)-p(\bar{v})-p^{\prime}(\bar{v}) V_{x}\right]\left[d^{\prime}(\tau) \bar{v}_{x}+\bar{v}_{t}\right] d x d \tau \\
\leq C \int_{0}^{t} \int_{0}^{\infty}\left|\hat{v}\left[d^{\prime}(\tau) \bar{v}_{x}+\bar{v}_{t}\right]\right| d x d \tau+C \int_{0}^{t} \int_{0}^{\infty}\left|V_{x}^{2}\left[d^{\prime}(\tau) \bar{v}_{x}+\bar{v}_{t}\right]\right| d x d \tau \\
\leq C \delta \int_{0}^{t} e^{-\alpha \tau} \int_{0}^{\infty} m_{0}(x) d x+C \delta \int_{0}^{t}(1+\tau)^{-\left(\gamma_{2}+\frac{1}{2}\right)}(\log (2+\tau))^{\frac{1}{2}}\left\|V_{x}(\tau)\right\|^{2} d \tau \\
\leq C \delta+C \delta \int_{0}^{t}\left\|V_{x}(\tau)\right\|^{2} d \tau, \quad \gamma_{2}>3 / 4
\end{aligned}
$$

and

$$
\begin{aligned}
& \int_{0}^{t} \int_{0}^{\infty}\left[-p\left(V_{x}+\bar{v}+\hat{v}\right) \hat{v}_{t}+\frac{1}{2} p^{\prime \prime}(\bar{v}) \frac{d \bar{v}}{d t} V_{x}^{2}\right] d x d \tau \\
& \leq C \delta \int_{0}^{t} e^{-\alpha \tau} \int_{0}^{\infty} m_{0}(x) d x \\
& \quad+C \delta \int_{0}^{t}\left\{(1+\tau)^{-\left(\gamma_{2}+1\right)}(\log (2+\tau))^{\frac{1}{2}}+(1+\tau)^{-\left(\gamma_{2}+\frac{3}{2}\right)}\right\}\left\|V_{x}(\tau)\right\|^{2} d \tau \\
& \leq C \delta+C \delta \int_{0}^{t}\left\|V_{x}(\tau)\right\|^{2} d \tau, \quad \gamma_{2}>3 / 4
\end{aligned}
$$


Due to $(3.22),(3.21),(3.24)-(3.26)$, and the integration by parts in $t$ for the term $V_{x t}$, we estimate $I_{3}$ as follows:

$$
\begin{aligned}
I_{3}= & \int_{0}^{t} \int_{0}^{\infty}\left[p\left(V_{x}+\bar{v}+\hat{v}\right)-p(\bar{v})-p^{\prime}(\bar{v}) V_{x}\right]\left(\lambda V_{x}+V_{x t}\right) d x d \tau \\
= & \int_{0}^{t} \int_{0}^{\infty}\left[p\left(V_{x}+\bar{v}+\hat{v}\right)-p(\bar{v})-p^{\prime}(\bar{v}) V_{x}\right] \lambda V_{x} d x d \tau \\
& +\int_{0}^{t} \frac{d}{d t} \int_{0}^{\infty}\left\{\int_{\bar{v}}^{V_{x}+\bar{v}+\hat{v}} p(s) d s-p(\bar{v}) V_{x}-\frac{1}{2} p^{\prime}(\bar{v}) V_{x}^{2}\right\} d x d \tau \\
& +\int_{0}^{t} \int_{0}^{\infty}-\left[p\left(V_{x}+\bar{v}+\hat{v}\right)-p(\bar{v})-p^{\prime}(\bar{v}) V_{x}\right] \frac{d}{d t} \bar{v} d x d \tau \\
& +\int_{0}^{t} \int_{0}^{\infty}\left[-p\left(V_{x}+\bar{v}+\hat{v}\right) \hat{v}_{t}+\frac{1}{2} p^{\prime \prime}(\bar{v}) \frac{d \bar{v}}{d t} V_{x}^{2}\right] d x d \tau \\
\leq & C \delta N(t)+C N(t) \int_{0}^{t}\left\|V_{x}(\tau)\right\|^{2} d \tau \\
& +\left.C \int_{0}^{\infty}\left\{\int_{\bar{v}}^{V_{x}+\bar{v}+\hat{v}} p(s) d s-p(\bar{v}) V_{x}-\frac{1}{2} p^{\prime}(\bar{v}) V_{x}^{2}\right\} d x\right|_{\tau=0} ^{t} \\
& +C \delta+C[\delta+N(t)] \int_{0}^{t}\left\|V_{x}(\tau)\right\|^{2} d \tau \\
\leq & C N(t)\left\|V_{x}(t)\right\|^{2}+C[\delta+N(t)] \int_{0}^{t}\left\|V_{x}(\tau)\right\|^{2} d \tau \\
& +C\left\|V_{0}\right\|_{1}^{2}+C(1+N(t)) \delta .
\end{aligned}
$$

If we combine (3.19), (3.20), and (3.27) with (3.16), we obtain

$$
\begin{aligned}
& \int_{0}^{t} \int_{0}^{\infty} f_{1} \cdot\left(\lambda V+V_{t}\right) d x d \tau \\
& \quad \leq C N(t)\left\|V_{x}(t)\right\|^{2}+C[\delta+N(t)] \int_{0}^{t}\left\|V_{x}(\tau)\right\|^{2} d \tau \\
& \quad+C\left\|V_{0}\right\|_{1}^{2}+C(1+N(t)) \delta
\end{aligned}
$$

In order to estimate the second part $f_{2}$, of the nonlinear term $F$, we notice that the terms $d^{\prime \prime}(t)\left[v_{+}-\bar{v}(x+d(t), t)\right], d^{\prime}(t)^{2} \bar{v}_{x}(x+d(t), t)$, and $d^{\prime}(t) \bar{v}_{t}(x+d(t), t)$ have a faster timedecay with respect to the last term $\alpha d^{\prime}(t)\left[v_{+}-\bar{v}(x+d(t), t)\right]$; then we restrict ourselves to analyze this last term. Since of $d(t)>0,(1+t)^{5 / 4}\left|V_{t}\right|$, one has $(1+t)^{1 / 4}|V| \leq C N(t)$ 
and (1.13), then it follows that

$$
\begin{aligned}
\mid \int_{0}^{t} & \int_{0}^{\infty} \alpha d^{\prime}(\tau)\left[v_{+}-\bar{v}(x+d(\tau), \tau)\right]\left(\lambda V+V_{t}\right) d x d \tau \mid \\
\leq & \alpha C \delta N(t) \int_{0}^{t} \int_{0}^{\infty} d^{\prime}(\tau) \exp \left(-\alpha c_{0}\left(\frac{x+d(\tau)}{\sqrt{\tau+1}}\right)^{2}\right)\left[(1+\tau)^{-1 / 4}+(1+\tau)^{-5 / 4}\right] d x d \tau \\
\leq & \alpha C \delta N(t) \int_{0}^{t}\left[(1+\tau)^{-1 / 4}+(1+\tau)^{-5 / 4}\right] \sqrt{\tau+1} d^{\prime}(\tau) \exp \left(-\alpha c_{0}\left(\frac{d(\tau)}{\sqrt{\tau+1}}\right)^{2}\right) \\
& \cdot \int_{0}^{\infty} \exp \left(-\alpha c_{0}\left(\frac{x}{\sqrt{\tau+1}}\right)^{2}\right) d \frac{x}{\sqrt{\tau+1}} d \tau \\
\leq & \alpha C \delta N(t) \int_{0}^{t}\left[(1+\tau)^{-1 / 4}+(1+\tau)^{-5 / 4}\right](1+\tau)^{-\gamma_{2}}(\log (2+\tau))^{1 / 2} d \tau \\
\leq & C \delta N(t), \quad \gamma_{2}>3 / 4
\end{aligned}
$$

Therefore, the integration on $f_{2}$ can be controlled in a similar way:

$$
\left|\int_{0}^{t} \int_{0}^{\infty} f_{2} \cdot\left(\lambda V+V_{t}\right) d x d \tau\right| \leq C \delta N(t) .
$$

Letting $N(t) \ll 1$ and applying (3.28) and (3.30) to (3.12) implies (3.2).

LEMMA 3.2. It follows that

$$
(1+t)\left\|\left(V_{x}, V_{t}\right)(t)\right\|^{2}+\int_{0}^{t}(1+\tau)\left\|V_{t}(\tau)\right\|^{2} d \tau \leq C\left(\left\|\left(V_{0}, V_{0, x}, V_{1}\right)\right\|^{2}+\delta\right),
$$

provided $N(T)+\delta \ll 1$.

Proof. Multiplying (1.37) by $(1+t) V_{t}$ and integrating it over $\mathbb{R}_{+}$with respect to $x$, we have

$$
\begin{aligned}
\frac{1}{2} \frac{d}{d t}\{ & \left.(1+t) \int_{0}^{t}\left(V_{t}^{2}-p^{\prime}(\bar{v}) V_{x}^{2}\right) d x\right\}+\alpha(1+t) \int_{0}^{\infty} V_{t}^{2} d x \\
= & \frac{1}{2} \int_{0}^{\infty}\left(V_{t}^{2}-p^{\prime}(\bar{v}) V_{x}^{2}\right) d x+\left.(1+t)\left(p^{\prime}(\bar{v}) V_{x} V_{t}\right)\right|_{x=0} \\
& -\int_{0}^{\infty}\left[\frac{p^{\prime \prime}(\bar{v})}{2}(1+t)\left(\bar{v}_{t}+d^{\prime}(t) \bar{v}_{x}\right) V_{x}^{2}\right] d x+\int_{0}^{\infty}(1+t) F V_{t} d x
\end{aligned}
$$

Because of the boundary decays, the formulas (3.11), (3.15) and $\gamma_{1}, \gamma_{3}>3 / 4$ yield

$$
\begin{aligned}
& \left|\int_{0}^{t}(1+\tau)\left(p^{\prime}(\bar{v}) V_{x} V_{t}\right)\right|_{x=0} d \tau \mid \\
& \quad \leq C \delta N(t) \int_{0}^{t}(1+\tau)(1+\tau)^{-\gamma_{3}}(1+\tau)^{-5 / 4} d \tau \\
& \quad \leq C \delta N(t)
\end{aligned}
$$


and

$$
\begin{aligned}
& \left|\int_{0}^{t}(1+\tau)\left[\left(p\left(V_{x}+\bar{v}+\hat{v}\right)-p(\bar{v})-p^{\prime}(\bar{v}) V_{x}\right) V_{t}\right]\right|_{x=0} d \tau \mid \\
& \quad \leq C \delta \int_{0}^{t}(1+\tau)(1+\tau)^{-\left(2 \gamma_{1}+\frac{5}{4}\right)} d \tau \\
& \quad \leq C \delta N(t) .
\end{aligned}
$$

Since $\left|\bar{v}_{t}(x+d(t), t)\right|=O(1)(1+t)^{-\left(\frac{3}{2}+\gamma_{2}\right)}$ and $\left|d^{\prime}(t) \bar{v}_{x}\right|=O(1)(1+t)^{-\left(1+\gamma_{2}\right)}(\log (2+t))^{\frac{1}{2}}$ for all $x \in \mathbb{R}_{+}, \gamma_{2}>3 / 4$, see (2.3), (2.4) and (1.11)-(1.13), by using the energy estimate (3.2), we get

$$
\left|\int_{0}^{\infty}\left[\frac{p^{\prime \prime}(\bar{v})}{2}(1+t)\left(\bar{v}_{t}+d^{\prime}(t) \bar{v}_{x}\right) V_{x}^{2}\right] d x\right| \leq C \int_{0}^{t}\left\|V_{x}(\tau)\right\|^{2} d \tau \leq C\left(\left\|\left(V_{0}, V_{0, x}, V_{1}\right)\right\|^{2}+\delta\right) .
$$

By using the estimate (3.29) for the slowest decay term for $\gamma_{2}>3 / 4$, one has

$$
\begin{aligned}
& \left|\int_{0}^{t} \int_{0}^{\infty}(1+\tau) \alpha d^{\prime}(\tau)\left[v_{+}-\bar{v}(x+d(\tau), \tau)\right] V_{t} d x d \tau\right| \\
& \quad \leq \alpha C \delta N(t) \int_{0}^{t}(1+\tau)(1+\tau)^{-\gamma_{2}}(\log (2+\tau))^{1 / 2}(1+\tau)^{-5 / 4} d \tau \\
& \quad=\alpha C \delta N(t) \int_{0}^{t}(1+\tau)^{-\left(\gamma_{2}+\frac{1}{4}\right)}(\log (2+\tau))^{1 / 2} d \tau \\
& \quad \leq C \delta N(t) .
\end{aligned}
$$

Furthermore, by the boundary integral (3.34), a similar calculation to (3.28) and (3.30) yields

$$
\begin{aligned}
& \int_{0}^{t} \int_{0}^{\infty}(1+\tau) F V_{t} d x d \tau=\int_{0}^{t} \int_{0}^{\infty}(1+\tau)\left(f_{1}+f_{2}\right) V_{t} d x d \tau \\
& \leq C N(t)(1+t)\left\|V_{x}(t)\right\|^{2}+C[\delta+N(t)] \int_{0}^{t}\left\|V_{x}(\tau)\right\|^{2} d \tau \\
& \quad+C\left\|V_{0}\right\|_{1}^{2}+C(1+N(t)) \delta .
\end{aligned}
$$

Thus, integrating (3.32) over $[0, t]$ and using (3.33)-(3.37) and the basic estimate (3.2), we prove (3.31) provided that $N(t)+\delta \ll 1$.

Step 2. The decay rate for $V_{x x}$ and $V_{x t}$. Let us differentiate (1.37) in $x$ and multiply the resulting equation by $V_{x t}$. Then by integrating it over $[0,+\infty)$ with respect to $x$, we get

$$
\begin{aligned}
& \frac{1}{2} \frac{d}{d t} \int_{0}^{\infty}\left(V_{x t}^{2}-p^{\prime}(\bar{v}) V_{x x}^{2}\right) d x+\frac{\alpha}{4} \int_{0}^{\infty} V_{x t}^{2} d x \\
& \leq \frac{1}{2} \frac{d}{d t} \int_{0}^{\infty}\left(p^{\prime}\left(V_{x}+\bar{v}+\hat{v}\right)-p^{\prime}(\bar{v})\right) V_{x x}^{2} d x+\left.\left(\left(p^{\prime}(\bar{v})\right) V_{x}\right)_{x} V_{x t}\right|_{x=0} \\
& \quad+C(N(t)+\delta)\left[(1+t)^{-2} \int_{0}^{\infty} V_{x}^{2} d x+(1+t)^{-1} \int_{0}^{\infty} V_{x x}^{2} d x\right] \\
& \quad+C \delta(1+t)^{-\left(\frac{9}{4}+\gamma_{3}\right)}(\log (2+t))^{\frac{1}{2}}
\end{aligned}
$$


Here we used (1.8), (1.11)-(1.13), and the decay estimates (2.6) for the diffusion waves. Note that $\frac{9}{4}+\gamma_{3}>3$, since $\gamma_{3}=\min \left\{\gamma_{1}, \gamma_{2}\right\}>3 / 4$.

From (3.9) and (2.3), (2.4), and the inequality $(1+t)^{5 / 4}\left|V_{x x}(x, t)\right| \leq C N(t)$, the boundary decay can be estimated as follows:

$$
\begin{aligned}
& \left|\left(\left(p^{\prime}(\bar{v})\right) V_{x}\right)_{x} V_{x t}\right|_{x=0} \mid \\
& \quad=\left|\left(p^{\prime \prime}(\bar{v}) \bar{v}_{x} G(t)+p^{\prime}(\bar{v}) V_{x x}\right)(0, t) G^{\prime}(t)\right| \\
& \quad \leq C \delta\left[(1+t)^{-\left(\frac{1}{2}+\gamma_{1}\right)}+N(t)(1+t)^{-5 / 4}\right](1+t)^{-\left(1+\gamma_{1}\right)} \\
& \quad \leq C \delta(1+t)^{-\left(\frac{9}{4}+\gamma_{1}\right)}
\end{aligned}
$$

where $\frac{9}{4}+\gamma_{1}>3$, since $\gamma_{1}>\frac{3}{4}$.

On the other hand, by differentiating (1.37) in $x$, by multiplying the resulting equation by $V_{x}$, and by integrating it over $[0,+\infty)$ with respect to $x$, we obtain

$$
\begin{aligned}
\frac{d}{d t} \int_{0}^{\infty} & \left(\frac{\alpha}{2} V_{x}^{2}+V_{x} V_{x t}\right) d x-\int_{0}^{\infty} V_{x t}^{2} d x+\int_{0}^{\infty}\left(-p^{\prime}(\bar{v})\right) V_{x x}^{2} d x \\
\leq & \left.\left(p^{\prime}(\bar{v}) V_{x}\right)_{x} V_{x}\right|_{x=0}+C \delta N(t)(1+t)^{-1} \int_{0}^{\infty} V_{x}^{2} d x \\
& +C \delta(1+t)^{-\left(\frac{5}{4}+\gamma_{3}\right)}(\log (2+t))^{\frac{1}{2}}
\end{aligned}
$$

where $\frac{5}{4}+\gamma_{3}>2$ since $\gamma_{3}=\min \left\{\gamma_{1}, \gamma_{2}\right\}>\frac{3}{4}$. As shown in (3.39), we also have

$$
\left|\left(\left(p^{\prime}(\bar{v})\right) V_{x}\right)_{x} V_{x}\right|_{x=0} \mid \leq C \delta(1+t)^{-\left(\frac{5}{4}+\gamma_{1}\right)}
$$

where $\frac{5}{4}+\gamma_{1}>2$ since $\gamma_{1}>\frac{3}{4}$.

By $(3.38)+\lambda \times(3.40)$ for $0<\lambda \ll 1$, integrating it over $[0, t]$, we have

LEMMA 3.3. It follows that

$$
\left\|\left(V_{x}, V_{x x}, V_{x t}\right)(t)\right\|^{2}+\int_{0}^{t}\left\|\left(V_{x x}, V_{x t}\right)(\tau)\right\|^{2} d \tau \leq C\left(\left\|\left(V_{0, x}, V_{0, x x}, V_{1, x}\right)\right\|^{2}+\delta\right)
$$

provided $N(T)+\delta \ll 1$.

By multiplying $(3.38)+\lambda \times(3.40)$ by $(1+t)$, and by using $(3.39),(3.41)$, and the inequality

$$
\int_{0}^{t}(1+\tau)(1+\tau)^{-\left(\frac{5}{4}+\gamma_{3}\right)}(\log (2+\tau))^{\frac{1}{2}} d \tau \leq C,
$$

since $\gamma_{3}>3 / 4$, by virtue of Lemmas 3.1 and 3.3 , we proved

LEMMA 3.4. It follows that

$$
(1+t)\left\|\left(V_{x}, V_{x x}, V_{x t}\right)(t)\right\|^{2}+\int_{0}^{t}(1+\tau)\left\|\left(V_{x t}, V_{x x}\right)(\tau)\right\|^{2} d \tau \leq C\left(\left\|V_{0}\right\|_{2}^{2}+\left\|V_{1}\right\|_{1}^{2}+\delta\right)
$$

provided $N(T)+\delta \ll 1$ 
Furthermore, by multiplying $(3.38)$ by $(1+t)^{2}$ and integrating it over $[0, t]$, thanks to (3.39) and Lemmas 3.1-3.4, as well as the fact

$$
\int_{0}^{t}(1+\tau)^{2}(1+\tau)^{-\left(\frac{9}{4}+\gamma_{3}\right)}(\log (2+\tau))^{\frac{1}{2}} d \tau \leq C
$$

since $\gamma_{3}>\frac{3}{4}$, we proved

LEMMA 3.5. It follows that

$$
\begin{aligned}
& (1+t)^{2}\left\|\left(V_{x x}, V_{x t}\right)(t)\right\|^{2}+\int_{0}^{t}\left[(1+\tau)\left\|V_{x x}(\tau)\right\|^{2}+(1+\tau)^{2}\left\|V_{x t}(\tau)\right\|^{2}\right] d \tau \\
& \leq C\left(\left\|V_{0}\right\|_{2}^{2}+\left\|V_{1}\right\|_{1}^{2}+\delta\right)
\end{aligned}
$$

provided $N(T)+\delta \ll 1$

Step 3. The decay rate for $V_{x x x}$ and $V_{x x t}$. First, we are going to prove the boundary estimate in the higher-order case. From (1.37), that is,

$$
V_{x t t}+\alpha V_{x t}+\left(p\left(V_{x}+\bar{v}+\hat{v}\right)-p(\bar{v})\right)_{x x}=-\frac{d}{d t} \bar{u}_{x}+f_{2 x}
$$

which implies

$$
\begin{aligned}
V_{x x x}=p^{\prime}\left(V_{x}+\bar{v}+\hat{v}\right)^{-1} & \left\{-V_{x t t}-\alpha V_{x t}-p^{\prime}\left(V_{x}+\bar{v}+\hat{v}\right)\left(\bar{v}_{x x}+\hat{v}_{x x}\right)\right. \\
& \left.+p^{\prime \prime}\left(V_{x}+\bar{v}+\hat{v}\right)\left(V_{x x}+\bar{v}_{x}+\hat{v}_{x}\right)^{2}+p(\bar{v})_{x x}-\frac{d}{d t} \bar{u}_{x}+f_{2 x}\right\}
\end{aligned}
$$

then (3.47) and $\left.V_{x}\right|_{x=0}=G(t),\left.\hat{v}\right|_{x=0}=0$ gives us

$$
\begin{aligned}
\left.V_{x x x}\right|_{x=0}=p^{\prime}\left(G(t)+\left.\bar{v}\right|_{x=0}\right)^{-1}\{ & -G^{\prime \prime}(t)-\alpha G^{\prime}(t) \\
& -\left.p^{\prime}\left(G(t)+\left.\bar{v}\right|_{x=0}\right)\left(\bar{v}_{x x}+\hat{v}_{x x}\right)\right|_{x=0} \\
& +p^{\prime \prime}\left(G(t)+\left.\bar{v}\right|_{x=0}\right)\left(\left.V_{x x}\right|_{x=0}+\left.\left(\bar{v}_{x}+\hat{v}_{x}\right)\right|_{x=0}\right)^{2} \\
& \left.+\left.p(\bar{v})_{x x}\right|_{x=0}-\left.\frac{d}{d t} \bar{u}_{x}\right|_{x=0}+\left.f_{2 x}\right|_{x=0}\right\}
\end{aligned}
$$

By making use of (3.48), (3.9), (2.3)-(2.5), (1.12), and (1.13), as well as $\gamma_{1}, \gamma_{2}>3 / 4$, and by integrating by parts with respect to $t$, thanks to the inequality

$$
(1+t)^{3 / 4}\left|V_{x}(0, t)\right|+(1+t)^{5 / 4}\left|V_{x x}(0, t)\right|+(1+t)^{7 / 4}\left|V_{x t}(0, t)\right| \leq C N(t)
$$


we estimate the boundary integral as follows:

$$
\begin{aligned}
\mid \int_{0}^{t}(1+ & \tau)\left.^{3}\left(\left(p^{\prime}(\bar{v}) V_{x}\right)_{x x} V_{x x t}\right)\right|_{x=0} d \tau \mid \\
\leq & C \delta+C \delta N(t)(1+t)^{-\left(\gamma_{3}-\frac{3}{4}\right)}(\log (2+t))^{\frac{1}{2}} \\
& +C \delta N(t)^{2}(1+t)^{-\gamma_{3}}(\log (2+t))^{\frac{1}{2}}+C \delta N(t)^{3}(1+t)^{-\frac{3}{4}} \\
& +C \delta N(t) \int_{0}^{t}(1+\tau)^{-\left(\gamma_{3}+\frac{1}{4}\right)}(\log (2+\tau))^{\frac{1}{2}} d \tau \\
& +C \delta N(t)^{2} \int_{0}^{t}(1+\tau)^{-\left(\gamma_{3}+1\right)}(\log (2+\tau))^{\frac{1}{2}} d \tau+C \delta N(t)^{3} \int_{0}^{t}(1+\tau)^{-\left(\gamma_{3}+\frac{5}{4}\right)} d \tau \\
\leq & C \delta, \quad \gamma_{3}=\min \left\{\gamma_{1}, \gamma_{2}\right\}>3 / 4
\end{aligned}
$$

provided $N(t) \ll 1$.

Differentiating (1.37) twice in $x$ and multiplying it by $(1+t)^{3} V_{x x t}$, by procedures similar to Step 2 and by using the estimate (3.49), we prove

LEMMA 3.6. It follows that

$$
\begin{aligned}
& (1+t)^{3}\left\|\left(V_{x x x}, V_{x x t}\right)(t)\right\|^{2}+\int_{0}^{t}\left[(1+\tau)^{2}\left\|V_{x x x}(\tau)\right\|^{2}+(1+\tau)^{3}\left\|V_{x x t}(\tau)\right\|^{2}\right] d \tau \\
& \leq C\left(\|\left(V_{0}\left\|_{3}^{2}+\right\| V_{1} \|_{2}^{2}+\delta\right)\right.
\end{aligned}
$$

provided $N(T)+\delta \ll 1$.

Step 4. The decay rate for $V_{t}$ and $V_{x t}$ and $V_{t t}$. By differentiating (1.37) in $t$ it follows that

$$
L(V)_{t}:=V_{t t t}+\alpha V_{t t}+\left(p^{\prime}(\bar{v}) V_{x}\right)_{x t}=F_{t} .
$$

By multiplying it by $V_{t t}$ and by integrating, with respect to $x$, the resulting identity on $[0,+\infty)$, we get

$$
\int_{0}^{\infty} V_{t t} \cdot L(V)_{t} d x=\int_{0}^{\infty} V_{t t} \cdot F_{t} d x .
$$

Hence, by a straightforward computation, we get

$$
\begin{aligned}
& \frac{1}{2} \frac{d}{d t} \int_{0}^{\infty}\left(V_{t t}^{2}-p^{\prime}(\bar{v}) V_{x t}^{2}\right) d x+\frac{\alpha}{4} \int_{0}^{\infty} V_{t t}^{2} d x \\
& \leq \frac{1}{2} \frac{d}{d t} \int_{0}^{\infty}\left(p^{\prime}\left(V_{x}+\bar{v}+\hat{v}\right)-p^{\prime}(\bar{v})\right) V_{x t}^{2} d x+\left.\left(\left(p^{\prime}(\bar{v})\right) V_{x}\right)_{t} V_{t t}\right|_{x=0} \\
&+C(N(t)+\delta)\left[(1+t)^{-3} \int_{0}^{\infty} V_{x}^{2} d x+(1+t)^{-2} \int_{0}^{\infty} V_{x x}^{2} d x\right] \\
&+C \delta(1+t)^{-\left(\frac{13}{4}+\gamma_{3}\right)}(\log (2+t))^{\frac{1}{2}}
\end{aligned}
$$

Thanks to the inequality

$$
(1+t)^{5 / 4}\left|V_{t}(0, t)\right| \leq C N(t),
$$


and $(2.3),(2.4),(3.9)$, and (1.12), by integrating by parts in $t$, we estimate the boundary integral as follows:

$$
\begin{aligned}
&\left|\int_{0}^{t}(1+\tau)^{3}\left(\left(p^{\prime}(\bar{v})\right) V_{x}\right)_{t} V_{t t}\right|_{x=0} d \tau \mid \\
&=\left|\int_{0}^{t}(1+\tau)^{3}\left(p^{\prime}(\bar{v}(d(\tau), \tau)) G^{\prime}(\tau)+p^{\prime \prime}(\bar{v}(d(\tau), \tau)) \bar{v}_{t}(d(\tau), \tau)\right) V_{t t}(0, \tau) d \tau\right| \\
&=\left|(1+\tau)^{3}\left(p^{\prime}(\bar{v}(d(\tau), \tau)) G^{\prime}(\tau)+p^{\prime \prime}(\bar{v}(d(\tau), \tau)) \bar{v}_{t}(d(\tau), \tau)\right) V_{t}(0, \tau)\right|_{\tau=0}^{t} \\
&-3 \int_{0}^{t}(1+\tau)^{2}\left(p^{\prime}(\bar{v}(d(\tau), \tau)) G^{\prime}(\tau)+p^{\prime \prime}(\bar{v}(d(\tau), \tau)) \bar{v}_{t}(d(\tau), \tau)\right) V_{t}(0, \tau) d \tau \\
&-\int_{0}^{t}(1+\tau)^{3}\left(p^{\prime}(\bar{v}(d(\tau), \tau)) G^{\prime}(\tau)+p^{\prime \prime}(\bar{v}(d(\tau), \tau)) \bar{v}_{t}(d(\tau), \tau)\right)_{t} V_{t}(0, \tau) d \tau \mid \\
& \leq C \delta N(t)\left(1+(1+t)^{-\left(\gamma_{3}-\frac{3}{4}\right)}+\int_{0}^{t}(1+\tau)^{-\left(1+\gamma_{3}-\frac{3}{4}\right)} d \tau\right) \\
& \leq C \delta N(t)
\end{aligned}
$$

provided $\gamma_{3}>3 / 4$ (see (3.9)).

On the other hand, by multiplying $(3.51)$ by $V_{t}$ we get

$$
\begin{aligned}
\frac{d}{d t} \int_{0}^{\infty} & \left(\frac{\alpha}{2} V_{t}^{2}+V_{t} V_{t t}\right) d x-\int_{0}^{\infty} V_{t t}^{2} d x+\int_{0}^{\infty}\left(-p^{\prime}(\bar{v})\right) V_{x t}^{2} d x \\
\leq & \left.\left(p^{\prime}(\bar{v}) V_{x}\right)_{t} V_{t}\right|_{x=0}+C \delta N(t)(1+t)^{-2} \int_{0}^{\infty} V_{x}^{2} d x \\
& +C \delta(1+t)^{-\left(\frac{9}{4}+\gamma_{3}\right)}(\log (2+t))^{\frac{1}{2}}
\end{aligned}
$$

As shown in (3.54), the boundary integral can also be estimated in the following way:

$$
\left|\int_{0}^{t}(1+\tau)^{2}\left(\left(p^{\prime}(\bar{v})\right) V_{x}\right)_{t} V_{t}\right|_{x=0} d \tau \mid \leq C \delta
$$

By taking $\int_{0}^{t}[\lambda \cdot(3.55)+(3.53)] d \tau, \int_{0}^{t}(1+\tau)[\lambda \cdot(3.55)+(3.53)] d \tau, \int_{0}^{t}(1+\tau)^{2}[\lambda \cdot(3.55)+$ (3.53)] $d \tau$ for $0<\lambda \ll 1$, respectively, and by using (3.54), (3.56), and (3.44), we have

LEMMA 3.7. It follows that

$$
\begin{gathered}
(1+t)^{2}\left\|\left(V_{t}, V_{t t}, V_{x t}\right)(t)\right\|^{2}+\int_{0}^{t}(1+\tau)^{2}\left\|\left(V_{t t}, V_{x t}\right)(\tau)\right\|^{2} d \tau \\
\leq C\left(\left\|V_{0}\right\|_{2}^{2}+\left\|V_{1}\right\|_{1}^{2}+\delta\right),
\end{gathered}
$$

provided $N(T)+\delta \ll 1$.

Finally, by using $\int_{0}^{t}(1+\tau)^{3}(3.53) d \tau$, by $(3.54),(3.57)$ and by

$$
\int_{0}^{t}(1+\tau)^{3}(1+\tau)^{-\left(\frac{13}{4}+\gamma_{3}\right)}(\log (2+\tau))^{\frac{1}{2}} d \tau \leq C,
$$

since $\gamma_{3}>3 / 4$, we obtain 
LEMMA 3.8. It follows that

$$
\begin{gathered}
(1+t)^{3}\left\|\left(V_{x t}, V_{t t}, V_{x t}\right)(t)\right\|^{2}+\int_{0}^{t}(1+\tau)^{3}\left\|V_{t t}(\tau)\right\|^{2} d \tau \\
\leq C\left(\left\|V_{0}\right\|_{2}^{2}+\left\|V_{1}\right\|_{1}^{2}+\delta\right)
\end{gathered}
$$

provided $N(T)+\delta \ll 1$.

A combination of the Lemmas 3.7 and 3.8 yields the optimal decay rates, namely,

LEMmA 3.9. Under the previous hypotheses, one has

$$
\begin{aligned}
(1+t)^{2} & \left\|V_{t}(t)\right\|^{2}+(1+t)^{3}\left\|\left(V_{t t}, V_{x t}\right)(t)\right\|^{2} \\
& +\int_{0}^{t}\left[(1+\tau)^{2}\left\|V_{x t}(\tau)\right\|^{2}+(1+\tau)^{3}\left\|V_{t t}(\tau)\right\|^{2}\right] d \tau \\
\leq & C\left(\left\|V_{0}\right\|_{2}^{2}+\left\|V_{1}\right\|_{1}^{2}+\delta\right),
\end{aligned}
$$

provided $N(T)+\delta \ll 1$.

Step 5. The decay rate for $V_{x x t}$ and $V_{x t t}$. By similar procedures as in Steps 2-4, since the boundary integration for the higher-order case can also be treated like Step 3, we can prove

LEMmA 3.10. Under the previous hypotheses, one has

$$
\begin{aligned}
(1+t)^{4} & \left(\left\|V_{x x t}(t)\right\|^{2}+\left\|V_{x t t}(t)\right\|^{2}\right) \\
& +\int_{0}^{t}\left[(1+\tau)^{3}\left\|V_{x x t}(\tau)\right\|^{2}+(1+\tau)^{4}\left\|V_{x t t}(\tau)\right\|^{2}\right] d \tau \\
\leq & C\left(\left\|\left(V_{0}\left\|_{3}^{2}+\right\| V_{1}\right)\right\|_{2}^{2}+\delta\right),
\end{aligned}
$$

provided $N(T)+\delta \ll 1$.

Combining Lemmas 3.2, 3.5, 3.9, and 3.10, we prove our estimate (2.8).

4. Concluding remarks. In this section, as concluding remarks, we are going to discuss two situations. One is the convergence in the special case $v_{+}=v_{-}$. Another one is the case of boundary layer on $u$.

In the case of $v_{+}=v_{-}$, we know that the equations (1.2) have the constant solutions $(\bar{v}, \bar{u})(x, t)=\left(v_{+}, 0\right)$. As shown in the Introduction, let

$$
\left\{\begin{array}{l}
V(x, t):=-\int_{x}^{\infty}\left[v(y, t)-v_{+}\right] d y, \quad(x, t) \in \mathbb{R}_{+} \times \mathbb{R}_{+} . \\
z(x, t):=u(x, t)
\end{array}\right.
$$

Then the IBVP (1.1), (1.6), and (1.7) is reduced to

$$
\begin{cases}V_{t}=z, & \\ z_{t}+p^{\prime}\left(v_{+}\right) V_{x x}+\alpha z=F_{1}, & (x, t) \in \mathbb{R}_{+} \times \mathbb{R}_{+}, \\ \left.(V, z)\right|_{t=0}=\left(-\int_{0}^{\infty}\left[v_{0}(y)-v_{+}\right] d y, u_{0}(x)\right):=\left(V_{0}, z_{0}\right)(x), & x \in \mathbb{R}_{+}, \\ \left.V_{x}\right|_{x=0}=g(t)-v_{+}=: G(t), & t \in \mathbb{R}_{+},\end{cases}
$$


where $F_{1}:=p\left(V_{x}+v_{+}\right)-p\left(v_{+}\right)-p^{\prime}\left(v_{+}\right) V_{x}$. Moreover, substituting $z=V_{t}$ into the second equation of (4.2), then (4.2) is rewritten as follows:

$$
\left\{\begin{array}{l}
V_{t t}+p^{\prime}\left(v_{+}\right) V_{x x}+\alpha V_{t}=F_{1}, \quad(x, t) \in \mathbb{R}_{+} \times \mathbb{R}_{+}, \\
\left.\left(V, V_{t}\right)\right|_{t=0}=\left(V_{0}, z_{0}\right)(x), \\
\left.V_{x}\right|_{x=0}=g(t)-v_{+}=: G(t) .
\end{array}\right.
$$

Since $V_{t t}$ decays faster than $V_{t}$, also hinted by the previous sections that the equation $V_{t t}+\alpha V_{t}+p^{\prime}\left(v_{+}\right) V_{x x}=F_{1}$ is essentially controlled by the part $\alpha V_{t}+p^{\prime}\left(v_{+}\right) V_{x x}$, so we denote it as follows:

$$
V_{t}+\frac{p^{\prime}\left(v_{+}\right)}{\alpha} V_{x x}=\frac{1}{\alpha}\left(F_{1}-V_{t t}\right)
$$

Thus, let us express formally the Neumann type IBVP (4.4) in the integral form

$$
V(x, t)=\int_{0}^{\infty} G(x, t ; y) V_{0}(y) d y+\frac{1}{\alpha} \int_{0}^{t} \int_{0}^{\infty} G(x, t-\tau ; y)\left(F_{1}-V_{t t}\right) d y d \tau
$$

where

$$
G(x, t ; y)=\frac{\sqrt{\alpha}}{\sqrt{-4 \pi p^{\prime}\left(v_{+}\right) t}}\left[e^{-\frac{\alpha(x-y)^{2}}{-4 p^{\prime}\left(v_{+}\right) t}}-e^{-\frac{\alpha(x+y)^{2}}{-4 p^{\prime}(v+) t}}\right]
$$

is the Green function of the heat equation in $\mathbb{R}_{+} \times \mathbb{R}_{+}$with Neumann boundary

$$
\begin{cases}u_{t}+\frac{p^{\prime}\left(v_{+}\right)}{\alpha} u_{x x}=0, & (x, t) \in \mathbb{R}_{+} \times \mathbb{R}_{+}, \\ \left.u\right|_{t=0}=u_{0}(x), & x \in \mathbb{R}_{+}, \\ \left.u_{x}\right|_{x=0}=u_{1}(t), & t \in \mathbb{R}_{+} .\end{cases}
$$

By the energy method used in the above section and a similar $L^{\infty}$-analysis as in Nishihara [19], as well as using (4.5), it is possible to state (without any proof) the following result.

REMARK 4.1. Suppose that

$$
G(t)=O(1)(1+t)^{-\gamma_{4}}, \quad \gamma_{4}>1,
$$

and $V_{0} \in H^{3}\left(\mathbb{R}_{+}\right) \cap L^{1}\left(\mathbb{R}_{+}\right), z_{0} \in H^{2}\left(\mathbb{R}_{+}\right) \cap L^{1}\left(\mathbb{R}_{+}\right)$, when $\left\|V_{0}\right\|_{3}+\left\|z_{0}\right\|_{2} \ll 1$, then the IBVP (4.3) has a unique global solution satisfying

$$
\left\|V_{x}(\cdot, t)\right\|_{L^{\infty}} \leq C(1+t)^{-1}, \quad\left\|V_{t}(\cdot, t)\right\|_{L^{\infty}} \leq C(1+t)^{-3 / 2} .
$$

The above decay rates are almost optimal in the $L^{\infty}$-sense, comparing with the corresponding Cauchy problem studied by Li [10], Zheng [21], and Nishihara [19].

Finally, we deal with the situation of boundary layer.

REMARK 4.2. If we put the boundary condition

$$
\left.u\right|_{x=0}=b(t)
$$

for equations (1.1), instead of the boundary condition (1.7), the corresponding convergence result is similar to Theorem 2.1 and Remark 4.1, under the natural restrictions on $b(t)$. 
Acknowledgments. This paper was completed when the second author visited the Dipartimento di Matematica Pura ed Applicata of the Universitá degli Studi di L'Aquila. He would like to express his thanks for their warm hospitality. The research of the first author was supported in part by the European TMR project under the Contract ERB-FMRX-CT96-0033. The research of the second author was supported in part by the JSPS Research Fellowship for Young Scientists, and the Ministry of Education of Japan Grant-in-Aid for JSPS under the Contract P-96169 and the CNR of Italy Progetto Speciale Fluido Dinamica.

\section{REFERENCES}

[1] I.-L. Chern, Multiple-mode diffusion waves for viscous nonstrictly hyperbolic conservation laws, Comm. Math. Phys. 138, 51-61 (1991)

[2] I.-L. Chern, Long-time effect of relaxation for hyperbolic conservation laws, Comm. Math. Phys. 172, 39-55 (1995)

[3] I.-L. Chern and T.-P. Liu, Convergence to diffusion waves of solutions for viscous conservation laws, Comm. Math. Phys. 110, 503-517 (1987), 120, 525-527 (1989)

[4] S. Claudi and F. R. Guarguaglini, Large time behaviour for the heat equation with absorption and convection, Adv. Appl. Math. 16, 377-401 (1995)

[5] S. Claudi, R. Natalini, and A. Tesei, Large time behaviour of a diffusion equation with strong convection, Ann. Scuola Norm. Sup. Pisa 3, 445-474 (1994)

[6] C. T. Van Duyn and L. A. Peletier, A class of similarity solutions of the nonlinear diffusion equation, Nonlinear Analysis T.M.A. 1, 223-233 (1977)

[7] L. Hsiao and T.-P. Liu, Convergence to nonlinear diffusion waves for solutions of a system of hyperbolic conservation laws with damping, Comm. Math. Phys. 143, 599-605 (1992)

[8] L. Hsiao and T.-P. Liu, Nonlinear diffusive phenomena of nonlinear hyperbolic systems, Chinese Ann. Math. Ser. B 14, 465-480 (1993)

[9] L. Hsiao and T. Luo, Nonlinear diffusion phenomena of solutions for the system of compressible adiabatic flow through porous media, J. Differential Equations 125, 329-365 (1996)

[10] T.-T. Li, Nonlinear heat conduction with finite speed of propagation, Proceedings of the China-Japan Symposium on Reaction-Diffusion Equations and Their Applications and Computational Aspects, T.-T. Li and M. Mimura, eds., World Sci. Pub., 1997

[11] T.-P. Liu and K. Nishihara, Asymptotic behavior for scalar viscous conservation laws with boundary effect, J. Differential Equations 133, 296-320 (1997)

[12] T.-P. Liu and S.-H. Yu, Propagation of a stationary shock layer in the presence of a boundary, Arch. Rational Mech. Anal. 139, 57-82 (1997)

[13] P. Marcati and A. Milani, The one-dimensional Darcy's law as the limit of a compressible Euler flow, J. Differential Equations 84, 129-147 (1990)

[14] P. Marcati, A. Milani, and P. Secchi, Singular convergence of weak solutions for a quasilinear nonhomogeneous hyperbolic system, Manuscripta Math. 60, 49-69 (1988)

[15] P. Marcati and B. Rubino, Hyperbolic to parabolic relaxation theory for quasilinear first order systems, J. Differential Equations 162, 359-399 (2000)

[16] A. Matsumura, Nonlinear hyperbolic equations and related topics in fluid dynamics, T. Nishida (ed.), Pub. Math. D'Orsay, 53-57 (1978)

[17] A. Matsumura and M. Mei, Convergence of travelling fronts of solutions of the p-system with viscosity in the presence of a boundary, Arch. Rational Mech. Anal. 146, 1-22 (1999)

[18] M. Mei and B. Rubino, Convergence to traveling waves with decay rates for solutions of the initial boundary problem to a nonconvex relaxation model, J. Differential Equations 159, 138-185 (1999)

[19] K. Nishihara, Convergence rates to nonlinear diffusion waves for solutions of system of hyperbolic conservation laws with damping, J. Differential Equations 131, 171-188 (1996)

[20] T. Nishida, Nonlinear hyperbolic equations and related topics in fluid dynamics, T. Nishida (ed.), Pub. Math. D'Orsay, 46-53 (1978)

[21] S. Zheng, Nonlinear Parabolic Equations and Hyperbolic-Parabolic Coupled Systems, Longmans, Green, NY, 1995 